\title{
COMPARISON OF COMMUTING ONE-PARAMETER GROUPS OF ISOMETRIES
}

\author{
OLA BRATTELI, HIDEKI KUROSE AND DEREK W. ROBINSON
}

\begin{abstract}
Let $\alpha, \beta$ be two commuting strongly continuous one-parameter groups of isometries on a Banach space $\mathscr{A}$ with generators $\delta_{\alpha}$ and $\delta_{\beta}$, and analytic elements $\mathscr{A}_{\omega}^{\alpha}, \mathscr{A}_{\omega}^{\beta}$, respectively. Then it is easy to show that if $\delta_{\alpha}$ is relatively bounded by $\delta_{\beta}$, then $\mathscr{A}_{\omega}^{\beta} \subseteq \mathscr{A}_{\omega}^{\alpha}$, and in this paper we establish the inverse implication for unitary one-parameter groups on Hilbert spaces and for one-parameter groups of ${ }^{*}$-automorphisms of abelian $C^{*}$-algebras. It is not known in general whether the inverse implication holds or not, but it does not hold for one-parameter semigroups of contractions.
\end{abstract}

\section{INTRODUCTION}

Our intention in this paper is to examine the extent to which the analytic elements determine a strongly continuous one-parameter group of isometries on a Banach space. For example, if $U, V$, are two one-parameter unitary groups on the Hilbert space $\mathscr{H}$ with analytic elements $\mathscr{H}_{\omega}(U), \mathscr{H}_{\omega}(V)$, and generators $H, K$, and if $U$ commutes with $V$ then we prove that $\mathscr{H}_{\omega}(U) \subseteq \mathscr{H}_{\omega}(V)$ if and only if $K$ is relatively bounded by $H$. Thus $\mathscr{H}_{\omega}(U)=\mathscr{H}_{\omega}(V)$ if and only if the generators are mutually relatively bounded. Similar results are proved for two continuous one-parameter groups of ${ }^{*}$-automorphisms of an abelian $C^{*}$-algebra.

We also establish by examples that the situation is quite different if one only compares $C^{\infty}$-elements, or if one considers contraction semigroups. In particular we construct two one-parameter groups of ${ }^{*}$-automorphisms of a simple $C^{*}$-algebra which commute and which have the same $C^{\infty}$-elements, but the generator of one group is not relatively bounded by the generator of the other. In addition we give two semigroups of contractions with the same analytic elements but with generators which are not comparable.

\section{GeNERAL GROUPS OF ISOMETRIES}

If $\alpha$ is a strongly continuous one-parameter group of isometries on a Banach space $\mathfrak{A}$, with generator $\delta_{\alpha}$, one many associate various classes of smooth

Received by the editors June 23, 1988 and, in revised form, October 17, 1988.

1980 Mathematics Subject Classification (1985 Revision). Primary 35P15; Secondary 35J20, 35J25, 35B05. 
elements with $(\mathfrak{A}, \alpha)$. The simplest such class is $\mathfrak{A}_{1}^{\alpha}=D\left(\delta_{\alpha}\right)$, the domain of $\delta_{\alpha}$, but in this paper we will be more concerned with the following classes: the class $\mathfrak{A}_{\infty}^{\alpha}=\bigcap_{n=1}^{\infty} D\left(\delta_{\alpha}^{n}\right)$ of infinitely many times differentiable elements, the subclass $\mathfrak{A}_{\omega}^{\alpha} \subseteq \mathfrak{A}_{\infty}^{\alpha}$ of analytic elements, i.e., elements $x \in \mathfrak{A}_{\infty}^{\alpha}$ such that

$$
\limsup _{n \rightarrow \infty} \frac{1}{n}\left\|\delta_{\alpha}^{n} x\right\|^{1 / n}<\infty
$$

and finally the subclass $\mathfrak{A}_{F}^{\alpha} \subseteq \mathfrak{A}_{\omega}^{\alpha}$ of $G$-finite or geometric elements, i.e., elements $x \in \mathfrak{A}_{\infty}^{\alpha}$ such that

$$
\limsup _{n \rightarrow \infty}\left\|\delta_{\alpha}^{n} x\right\|^{1 / n}<\infty
$$

If $\mathfrak{A}^{\alpha}(K)$ is the Arveson spectral subspace of $\mathfrak{A}$ corresponding to the compact subset $K \subseteq \widehat{\mathbf{R}}=\mathbf{R}$, then $\mathfrak{A}_{F}^{\alpha}=\bigcup_{K} \mathfrak{A}^{\alpha}(K)$, where the union is over all compact subsets. (See [BR, B] for details.)

Most of the implications in the following proposition are both well known and straightforward. The implication $(2) \Rightarrow(3)$ has also been proved in greater generality in [GJ, N]. Our main purpose in formulating this proposition is the belief that all six conditions are equivalent. We will show that this is indeed the case in the context of Hilbert spaces in $\S 3$, and in the context of groups of automorphisms on abelian $C^{*}$-algebras in $\S 4$. In $\S 5$ we show that the six conditions are not equivalent to $\mathfrak{A}_{\infty}^{\beta} \subseteq \mathfrak{A}_{\infty}^{\alpha}$, even under the special assumptions in $\S \S 3$ and 4. Finally we show in $\S 6$ that the results do not extend to oneparameter semigroups of contractions.

Proposition 2.1. Let $\alpha, \beta$ be two commuting strongly continuous one-parameter groups of isometries on a Banach space $\mathfrak{A}$ with generators $\delta_{\alpha}, \delta_{\beta}$, and let $\mathfrak{A}_{\infty}^{\alpha, \beta}=\mathfrak{A}_{\infty}^{\alpha} \cap \mathfrak{A}_{\infty}^{\beta}$

Consider the following six conditions:

(1) There exist constants $c, d \geq 0$ such that

$$
\mathfrak{A}^{\beta}([-L, L]) \subseteq \mathfrak{A}^{\alpha}([-c L-d, c L+d])
$$

for all $L \geq 0$.

(1) $)^{\prime}$ There exist constants $c, d \geq 0$ such that

$$
\limsup _{n \rightarrow \infty}\left\|\delta_{\alpha}^{n}(x)\right\|^{1 / n} \leq c \limsup _{n \rightarrow \infty}\left\|\delta_{\beta}^{n}(x)\right\|^{1 / n}+d
$$

for all $x \in \mathfrak{A}_{\infty}^{\alpha, \beta}$.

(2) $D\left(\delta_{\beta}\right) \subseteq D\left(\delta_{\alpha}\right)$.

(2) ${ }^{\prime}$ There exist constants $c, d \geq 0$ such that

$$
\left\|\delta_{\alpha}(x)\right\| \leq c\left\|\delta_{\beta}(x)\right\|+d\|x\|
$$

for all $x \in \mathfrak{A}_{\infty}^{\alpha, \beta}$.

(3) $\mathfrak{A}_{\omega}^{\beta} \subseteq \mathfrak{A}_{\omega}^{\alpha}$. 
(3) If $\lim \sup _{n \rightarrow \infty}(1 / n)\left\|\delta_{\beta}^{n}(x)\right\|^{1 / n}<\infty$ then

$$
\limsup _{n \rightarrow \infty} \frac{1}{n}\left\|\delta_{\alpha}^{n}(x)\right\|^{1 / n}<\infty,
$$

for all $x \in \mathfrak{A}_{\infty}^{\alpha, \beta}$.

The following implications are valid:

$$
\begin{aligned}
& (1) \Leftarrow(2) \Rightarrow \text { (3) } \\
& \begin{array}{ccc}
\sqrt{4} & \mathbb{1} & \mathbb{1} \\
(1)^{\prime} & (2)^{\prime} & (3)^{\prime}
\end{array}
\end{aligned}
$$

Furthermore, the constants $c, d$ occurring in (1) and (1)' can be taken to be the same, and if (2)' holds, then (1) holds with the same $c, d$.

Remark. We remark that conditions (2) and (2)' also imply the two equivalent conditions

(2n) $D\left(\delta_{\beta}^{n}\right) \subseteq D\left(\delta_{\alpha}^{n}\right)$, and

$(2 n)^{\prime}$ there exist constants $c, d \geq 0$ such that

$$
\left\|\delta_{\alpha}^{n}(x)\right\| \leq c\left\|\delta_{\beta}^{n}(x)\right\|+d\|x\|
$$

for all $x \in \mathfrak{A}_{\infty}^{\alpha, \beta}$. Here $n=2,3,4, \ldots$. This is clear from the estimates in the proof, and estimates of the form

$$
\left\|\delta^{m} x\right\| \leq K(n, m)\left(\left\|\delta^{n} x\right\|+\|x\|\right)
$$

which are valid for $m=0,1, \ldots, n$, and for $\delta=\delta_{\alpha}, \delta=\delta_{\beta}$, see [R1].

Also, if $R>0$ and

$$
\begin{aligned}
\mathfrak{A}_{\omega}^{\alpha}(R) & =\left\{x \in \mathfrak{A}_{\infty}^{\alpha} ; \text { radius of convergence of } \sum_{n=0}^{\infty} \frac{t^{n}}{n !}\left\|\delta_{\alpha}^{n}(x)\right\|\right. \\
& \text { is greater than } R\} \\
& =\left\{x \in \mathfrak{A}_{\infty}^{\alpha} ; \limsup _{n \rightarrow \infty} \frac{1}{n}\left\|\delta_{\alpha}^{n} x\right\|^{1 / n}<(e R)^{-1}\right\},
\end{aligned}
$$

then it follows from the proof that $(2)^{\prime}$ implies

$(3)^{\prime \prime} \mathfrak{A}_{\omega}^{\beta}(R) \subseteq \mathfrak{A}_{\omega}^{\alpha}(R / c \wedge 1 / d)$,

and since $\mathfrak{A}_{\omega}^{\beta}=\bigcup_{R>0} \mathfrak{A}_{\omega}^{\beta}(R)$ this again implies (3).

Proof. (3) $\Leftrightarrow(3)^{\prime}$ is trivial since $x$ is analytic for $\alpha$ if and only if

$$
\limsup _{n \rightarrow \infty} \frac{1}{n}\left\|\delta_{\alpha}^{n} x\right\|^{1 / n}<+\infty
$$

and $(2) \Leftrightarrow(2)^{\prime}$ is a consequence of Hørmander's closed graph theorem [Y, II.6, Theorem 2]. (Since $\alpha$ and $\beta$ commute, $\mathfrak{A}_{\infty}^{\alpha, \beta}$ is a joint core for $\delta_{\alpha}$ and $\delta_{\beta}$, see [BR, Corollary 3.1.7].) 
$(2)^{\prime} \Rightarrow(3)$. Since $\alpha$ and $\beta$ commute, we find

$$
\left\|\delta_{\alpha}^{n}(x)\right\| \leq \sum_{r=0}^{n}\left(\begin{array}{l}
n \\
r
\end{array}\right) c^{r} d^{n-r}\left\|\delta_{\beta}^{r}(x)\right\| .
$$

If $x \in \mathfrak{A}_{\omega}^{\beta}$, there exist constants $K, b$ such that $\left\|\delta_{\beta}^{r}(x)\right\| \leq K r ! b^{r}$ and so

$$
\begin{aligned}
\left\|\delta_{\alpha}^{n}(x)\right\| & \leq K \sum_{r=0}^{n} \frac{n !}{(n-r) !}(c b)^{r} d^{n-r} \\
& \leq K(n+1) !(c b \vee d)^{n} .
\end{aligned}
$$

Thus $x \in \mathfrak{A}_{\omega}^{\alpha}$.

For the remaining implications, we need the following.

Lemma 2.2. Let $\alpha$ be a strongly continuous one-parameter group of isometries on a Banach space $\mathfrak{A}$, with generator $\delta_{\alpha}$. Then the following conditions are equivalent for each $L \geq 0$ :

$$
\begin{gathered}
x \in \mathfrak{A}^{\alpha}([-L, L]), \\
\limsup _{n \rightarrow \infty}\left\|\delta_{\alpha}^{n}(x)\right\|^{1 / n} \leq L .
\end{gathered}
$$

Proof. $(2.1) \Rightarrow(2.2)$. For any $n$ we can write $x=\sum_{k=-n}^{n} x_{k}$ where the $\alpha$-spectrum of $x_{k}$ is contained in

$$
\left[\frac{k-1}{n} L, \frac{k+1}{n} L\right]
$$

by putting $x_{k}=\alpha_{f_{k}}(x)$ where

$$
\operatorname{supp} \hat{f}_{k} \subseteq\left[\frac{k-1}{n} L, \frac{k+1}{n} L\right]
$$

and $\sum_{k=-n}^{n} \hat{f}_{k}(r)=1$ if $r \in[-L, L]$. By [KR, Lemma 2.4] we have

$$
\left\|\left(\delta_{\alpha}-i \frac{k}{n} L\right)\left(x_{k}\right)\right\| \leq N \frac{1}{n}\left\|x_{k}\right\|
$$

where $N \geq 1$ is a universal constant. Hence,

$$
\left\|\delta_{\alpha}\left(x_{k}\right)\right\| \leq\left(\frac{|k|}{n} L+\frac{N}{n} L\right)\left\|x_{k}\right\| \leq\left(L+\frac{N}{n}\right)\left\|x_{k}\right\| .
$$

Now, since the powers $\delta_{\alpha}^{m}\left(x_{k}\right)$ all have $\alpha$-spectrum in

$$
\left[\frac{k-1}{n} L, \frac{k+1}{n} L\right]
$$

we may iterate the above estimate to obtain

$$
\left\|\delta_{\alpha}^{m}\left(x_{k}\right)\right\| \leq(L+N / n)^{m}\left\|x_{k}\right\| .
$$

Hence,

$$
\left\|\delta_{\alpha}^{m}(x)\right\| \leq K(n)(L+N / n)^{m}
$$


where $K(n)$ depends on $x$ and $n$. Thus

$$
\limsup _{m \rightarrow \infty}\left\|\delta_{\alpha}^{m}(x)\right\|^{1 / m}<L+N / n
$$

and since $n$ is arbitrary, (2.2) follows.

$(2.2) \Rightarrow(2.1)$. We show this by contradiction: If $x$ satisfies (2.2), but $x \in \mathfrak{A} \backslash \mathfrak{A}^{\alpha}([-L, L])$, then there exists an $a$ with $|a|>L$ such that $a \in \operatorname{Sp}_{\alpha}(x)$. Choose $\varepsilon>0$ such that $[-L, L] \cap[a-N \varepsilon, a+N \varepsilon]=\varnothing$ (where $N$ is the $N$ in Lemma 2.4 of [KR]), and choose $f \in L(\mathbf{R})$ such that $\hat{f}(a)=1$ and $\operatorname{supp} \hat{f} \subseteq[a-\varepsilon, a+\varepsilon]$. Then $y=\alpha_{f}(x) \neq 0$ and $\operatorname{Sp}_{\alpha}(y) \subseteq[a-\varepsilon, a+\varepsilon]$. Hence $\left\|\left(\delta_{\alpha}-i a\right)(y)\right\| \leq N \varepsilon\|y\|$ and, assuming $a>0,\left\|\delta_{\alpha} y\right\| \geq(a-N \varepsilon)\|y\|$. Iterating, we get $\left\|\delta_{\alpha}^{n} y\right\| \geq(a-N \varepsilon)^{n}\|y\|$ and hence

$$
\limsup _{n \rightarrow \infty}\left\|\delta_{\alpha}^{n}(y)\right\|^{1 / n} \geq a-N \varepsilon .
$$

On the other hand,

$$
\left\|\delta_{\alpha}^{n} y\right\|=\left\|\delta_{\alpha}^{n} \alpha_{f}(x)\right\|=\left\|\alpha_{f}\left(\delta_{\alpha}^{n}(x)\right)\right\| \leq|f|_{1}\left\|\delta_{\alpha}^{n}(x)\right\|,
$$

so

$$
\limsup _{n \rightarrow \infty}\left\|\delta_{\alpha}^{n}(y)\right\|^{1 / n} \leq \limsup _{n \rightarrow \infty}\left\|\delta_{\alpha}^{n}(x)\right\|^{1 / n} \leq L .
$$

But as $a-N \varepsilon>L$, this gives a contradiction and the implication $(2.2) \Rightarrow(2.1)$ is established.

Condition (1) of Proposition 2.1 can now be formulated as

$$
\limsup _{n \rightarrow \infty}\left\|\delta_{\beta}^{n}(x)\right\|^{1 / n} \leq L \Rightarrow \limsup _{n \rightarrow \infty}\left\|\delta_{\alpha}^{n}(x)\right\|^{1 / n} \leq c L+d
$$

and hence the equivalence of $(1)$ and $(1)^{\prime}$ is clear. We now prove $(2)^{\prime} \Rightarrow(1)^{\prime}$. From $(2)^{\prime}$ it follows by iteration that

$$
\left\|\delta_{\alpha}^{n} x\right\| \leq \sum_{r=0}^{n}\left(\begin{array}{l}
n \\
r
\end{array}\right) c^{r} d^{n-r}\left\|\delta_{\beta}^{r}(x)\right\| .
$$

Let $L=\limsup _{r \rightarrow \infty}\left\|\delta_{\beta}^{r}(x)\right\|^{1 / r}$. If $L=+\infty$ there is nothing to prove; if $L<+\infty$ there exists for each $\varepsilon>0$ a $K_{\varepsilon}$ such that $\left\|\delta_{\beta}^{r}(x)\right\| \leq K_{\varepsilon}(L+\varepsilon)^{r}$ for $r=0,1,2, \ldots$, and hence

$$
\left\|\delta_{\alpha}^{n} x\right\| \leq \sum_{r=0}^{n}\left(\begin{array}{l}
n \\
r
\end{array}\right) c^{r} d^{n-r} K_{\varepsilon}(L+\varepsilon)^{r}=K_{\varepsilon}(c(L+\varepsilon)+d)^{n} .
$$

Thus

$$
\limsup _{n \rightarrow \infty}\left\|\delta_{\alpha}^{n} x\right\|^{1 / n} \leq c(L+\varepsilon)+d
$$

but $\varepsilon$ was arbitrary so (1) ${ }^{\prime}$ holds.

We have thus established Proposition 2.1. 


\section{Hilbert SPACES}

Proposition 3.1. Adopt the assumptions of Proposition 2.1, but assume in addition that $\mathfrak{A}$ is a Hilbert space. Then conditions (1), (2) and (3) are all equivalent.

Remark. The technique used in the proof of $(1) \Rightarrow(2)^{\prime}$ also shows that conditions $(2 n)$ and $(2 n)^{\prime}$ in the remark after Proposition 2.1 are equivalent to (1), $(2)$ and $(3)$ in this setting.

Proof. Put $\delta_{\alpha}=i H_{\alpha}$ and $\delta_{\beta}=i H_{\beta}$, then $H_{\alpha}$ and $H_{\beta}$ are commuting selfadjoint operators. There is a measure space $(X, \mu)$ such that $H_{\alpha}$ and $H_{\beta}$ are represented by multiplication with real functions $h_{\alpha}$ and $h_{\beta}$ on $L^{2}(X, \mu)$. We have to prove $(1) \Rightarrow(2)^{\prime}$ and $(3) \Rightarrow(2)^{\prime}$.

$(1) \Rightarrow(2)^{\prime}$. Condition 1 means that

$$
\left|h_{\beta}(x)\right| \leq L \Rightarrow\left|h_{\alpha}(x)\right| \leq c L+d
$$

for $\mu$-almost all $x \in X$; hence $\left|h_{\alpha}(x)\right| \leq c\left|h_{\beta}(x)\right|+d$ for almost all $x \in X$, but then

$$
\begin{aligned}
\left\|H_{\alpha} \psi\right\|^{2} & =\int_{X}\left|h_{\alpha}(x) \psi(x)\right|^{2} d \mu(x) \leq \int_{X}\left|\left(c\left|h_{\beta}(x)\right|+d\right) \psi(x)\right|^{2} d \mu(x) \\
& =\left\|\left(c\left|H_{\beta}\right|+d\right) \psi\right\|^{2} \leq\left(c\left\|H_{\beta} \psi\right\|+d\|\psi\|\right)^{2}
\end{aligned}
$$

using the Cauchy-Schwarz inequality.

$(3) \Rightarrow(2)^{\prime}$. Assume that $(2)^{\prime}$ does not hold, i.e., that $\left|h_{\alpha}\right|$ is not linearly bounded by $\left|h_{\beta}\right|$. This means that

$$
\mu\left(\left\{x ;\left|h_{\alpha}(x)\right| \geq n\left(\left|h_{\beta}(x)\right|+1\right)\right\}\right)>0
$$

for all $n$. A simple argument then establishes that there exists a sequence $S_{n}$ of pairwise disjoint sets in $X$ such that $\mu_{n}=\mu\left(S_{n}\right)>0, \mu_{n}<\infty$, and

$$
S_{n} \subseteq\left\{x ;\left|h_{\alpha}(x)\right| \geq n\left(\left|h_{\beta}(x)\right|+1\right)\right\} .
$$

Now, note that $H_{\alpha}$ has the same analytic elements as $\left|H_{\alpha}\right|$, and $H_{\beta}$ the same as $\left|H_{\beta}\right|$, and since $e^{-t\left|H_{\alpha}\right|}$ and $e^{-t\left|H_{\beta}\right|}$ are holomorphic semigroups, it follows that

$$
\mathfrak{A}_{\omega}^{\beta}=\bigcup_{t>0} e^{-t\left|H_{\beta}\right|} \mathfrak{A} \quad \text { and } \quad \mathfrak{A}_{\omega}^{\alpha}=\bigcup_{t>0} e^{-t\left|H_{\alpha}\right|} \mathfrak{A},
$$

see [BGJR, Corollary 2.6].

We want to show that $\mathfrak{A}_{\omega}^{\beta} \nsubseteq \mathfrak{A}_{\omega}^{\alpha}$, i.e., that there exists a $\psi \in L^{2}(X, d \mu)$ such that $\psi$ is analytic for $H_{\beta}$ but not for $H_{\alpha}$. Define $\psi=e^{-\left|H_{\beta}\right|} \eta$ where

$$
\eta=\sum_{n=0}^{\infty} \frac{1}{n \sqrt{\mu_{n}}} \chi_{S_{n}}
$$

and $\chi_{S_{n}}$ is the characteristic function of $S_{n}$. Then

$$
\psi(x)=e^{-\left|h_{\beta}(x)\right|} \sum_{n=0}^{\infty} \frac{1}{n \sqrt{\mu_{n}}} \chi_{S_{n}}(x)
$$


is analytic for $H_{\beta}$. In order that $\psi$ shall be analytic for $H_{\alpha}$, there must exist a $\xi \in L^{2}(x, d \mu)$ and a $t>0$ s.t. $\psi=e^{-t\left|H_{\alpha}\right|} \xi$, i.e.,

$$
e^{-t\left|h_{\alpha}(x)\right|} \xi(x)=e^{-\left|h_{\beta}(x)\right|} \sum_{n=0}^{\infty} \frac{1}{n \sqrt{\mu_{n}}} \chi_{S_{n}}(x),
$$

i.e.,

$$
\xi(x)=\sum_{n=0}^{\infty} \frac{1}{n \sqrt{\mu_{n}}} e^{t\left|h_{\alpha}(x)\right|-\left|h_{\beta}(x)\right|} \chi_{S_{n}}(x) .
$$

But when $x \in S_{n},\left|h_{\alpha}(x)\right| \geq n\left(\left|h_{\beta}(x)\right|+1\right)$, so

$$
\xi(x) \geq \sum_{n=1}^{\infty} \frac{1}{n \sqrt{\mu_{n}}} e^{(t n-1)\left|h_{\beta}(x)\right|} e^{t n} \chi_{S_{n}}(x),
$$

and hence

$$
\|\xi\|_{2}^{2} \geq \sum_{n>1 / t} \frac{e^{2 t n}}{n^{2}}=+\infty .
$$

Thus, for no $t>0$ does there exist a $\xi \in \mathfrak{A}$ such that $\psi=e^{-t\left|h_{\alpha}\right|} \xi$, and thus $\psi \notin \mathfrak{A}_{\omega}^{\alpha}$. Hence $\mathfrak{A}_{\omega}^{\beta} \nsubseteq \mathfrak{A}_{\omega}^{\alpha}$, and $(3) \Rightarrow(2)^{\prime}$ is proved.

\section{Abelian $C^{*}$-algebras}

Proposition 4.1. Adopt the assumptions of Proposition 2.1, but assume in addition that $\mathfrak{A}$ is an abelian $C^{*}$-algebra and that $\alpha$ and $\beta$ are one-parameter groups of ${ }^{*}$-automorphisms. Then conditions (1), (2) and (3) are all equivalent, and in turn they are equivalent to the three conditions:

(1)" There exists a constant $c \geq 0$ such that

$$
\mathfrak{A}^{\beta}([-L, L]) \subseteq \mathfrak{A}^{\alpha}([-c L, c L])
$$

for all $L>0$.

$(2)^{\prime \prime}$ There exists a constant $c \geq 0$ such that $\left\|\delta_{\alpha}(x)\right\| \leq c\left\|\delta_{\beta}(x)\right\|$ for all $x \in \mathfrak{A}_{\infty}^{\alpha, \beta}$.

$(3)^{\prime \prime}$ If $S_{t}^{\beta}$ is the flow of homeomorphisms on the spectrum $\Omega$ of $\mathfrak{A}$ defined by $\beta$, there exists a continuous function $l$ on $\Omega \backslash \Omega_{0}$, where $\Omega_{0}$ is the fixed point for $S^{\beta}$, such that $l$ is constant on the $S^{\beta}$-orbits, $l$ is bounded and

$$
\left(\delta_{\alpha} f\right)= \begin{cases}l(\omega)\left(\delta_{\beta} f\right)(\omega) & \text { for } \omega \in \Omega \backslash \Omega_{0}, \\ 0 & \text { for } \omega \in \Omega_{0}\end{cases}
$$

for all $f \in \mathfrak{A}_{\infty}^{\alpha, \beta} \subseteq \mathfrak{A}=C_{0}(\Omega)$. 
Furthermore, the optimal values of $c$ in conditions (1), (1) $,(1)^{\prime \prime},(2)^{\prime}$ and $(2)^{\prime \prime}$ are all the same, and equal to

$$
\|l\|_{\infty}=\sup \left\{|l(\omega)| ; \omega \in \Omega \backslash \Omega_{0}\right\} .
$$

Remark. Using $\delta_{\alpha}^{n}=l^{n} \delta_{\beta}^{n}$ where the $l$ is defined by (4), one shows as in the proof that each of the conditions $(2 n)$ and $(2 n)^{\prime}$ in the remark after Proposition 2.1 also are equivalent to (1), (2) and (3).

Proof. Since clearly $(2)^{\prime \prime} \Rightarrow(2)^{\prime}$, we only have to prove that $(3) \Rightarrow(2)^{\prime \prime}$, $(1) \Rightarrow(1)^{\prime \prime} \Rightarrow(2)^{\prime \prime}$ and $(2) \Leftrightarrow(4)$.

(3) $\Rightarrow(2)^{\prime \prime}$. Assume that $\mathfrak{A}_{\omega}^{\beta} \subseteq \mathfrak{A}_{\omega}^{\alpha}$. If $\mathfrak{A}_{F}^{\beta}$ denotes the ${ }^{*}$-algebra of elements in $\mathfrak{A}$ with compact $\beta$-spectrum, then $\mathfrak{A}_{F}^{\beta} \subseteq \mathfrak{A}_{\omega}^{\beta} \subseteq \mathfrak{A}_{\omega}^{\alpha} \subseteq D\left(\delta_{\alpha}\right)$, so by [KR, Theorem 2.3] there exists a continuous function $l$ on $\Omega \backslash \Omega_{0}$, which is constant on $S^{\beta}$-orbits and bounded on sets of bounded frequency, such that

$$
\left(\delta_{\alpha} f\right)(\omega)=l(\omega)\left(\delta_{\beta} f\right)(\omega)
$$

for $f$ in the joint core $D\left(\delta_{\alpha}\right) \cap D\left(\delta_{\beta}\right)$ of $\delta_{\alpha}$ and $\delta_{\beta}$. From this relation it is immediate, using [BDGR, Lemma 2.7], that $\delta_{\alpha}$ is $\delta_{\beta}$-bounded if and only if $\|l\|_{\infty}=\sup \left\{\mid l(\omega) ! ; \omega \in \Omega \backslash \Omega_{0}\right\}$ is bounded, and then

$$
\left\|\delta_{\alpha} f\right\| \leq\|l\|_{\infty}\left\|\delta_{\beta} f\right\|
$$

is the best possible bound. This, incidentally, establishes $(2) \Leftrightarrow(4)$. To show that $\|l\|_{\infty}$ is finite we will use the following.

Lemma 4.2. Let $\delta$ be the generator of a one-parameter group of isometries on a Banach space $\mathfrak{A}$, and define

$$
|\delta|=\sqrt{-\delta^{2}}=\frac{1}{\pi} \int_{0}^{\infty} \lambda^{-1 / 2}\left(\lambda 1-\delta^{2}\right)^{-1}\left(-\delta^{2}\right) d \lambda,
$$

such that $|\delta|$ is the generator of the semigroup given by the Poisson kernel

$$
e^{-t|\delta|}=\frac{t}{\pi} \int_{-\infty}^{\infty} \frac{d s}{t^{2}+s^{2}} e^{s \delta} .
$$

Then an element $x \in \mathfrak{A}$ is analytic for $\delta$ if and only if it is analytic for $|\delta|$, and the two power series

$$
\sum_{n=0}^{\infty} \frac{t^{n}}{n !}\left\|\delta^{n} x\right\| \quad \text { and } \quad \sum_{n=0}^{\infty} \frac{t^{n}}{n !}\left\||\delta|^{n} x\right\|
$$

have the same radius of convergence.

Proof. It follows from [Y, IX.11] that $|\delta|$ is the generator of the semigroup $e^{-t|\delta|}$ given in the lemma, and that $|\delta|^{2}=-\delta^{2}$. Furthermore since $|\delta|$ and $\delta$ are dissipative it follows from [R1, Lemma 2.3] that

$$
\|\delta x\| \leq \varepsilon\left\|\delta^{2} x\right\|+\frac{2}{\varepsilon}\|x\| \text { and }\||\delta| x\| \leq \varepsilon\left\|\delta^{2} x\right\|+\frac{2}{\varepsilon}\|x\|
$$


for all $x \in D\left(\delta^{2}\right)$ and all $\varepsilon>0$. Hence we find $\left\|\delta^{2 m} x\right\|=\left\||\delta|^{2 m} x\right\|$ and

$$
\begin{gathered}
\left\|\delta^{2 m+1} x\right\| \leq\left\||\delta|^{2 m+2} x\right\|+2\left\||\delta|^{2 m} x\right\|, \\
\left\||\delta|^{2 m+1} x\right\| \leq\left\|\delta^{2 m+2} x\right\|+2\left\|\delta^{2 m} x\right\|
\end{gathered}
$$

for $m=0,1,2, \ldots$. The assertion of the lemma is immediate from these estimates.

We now return to the proof of $(3) \Rightarrow(2)^{\prime \prime}$ of Proposition 4.1. Assume ad absurdum that $l$ is unbounded, i.e., there exists a sequence $\omega_{n} \in \Omega \backslash \Omega_{0}$ such that $\left|l\left(\omega_{n}\right)\right| \rightarrow \infty$, and we may assume that $\omega_{n} \rightarrow\left|l\left(\omega_{n}\right)\right|$ is strictly monotone. Put

$$
U_{n}=\left\{\omega \in \Omega \backslash \Omega_{0} ; \frac{1}{2}\left(\left|l\left(\omega_{n}\right)\right|+\left|l\left(\omega_{n-1}\right)\right|\right)<|l(\omega)|<\frac{1}{2}\left(\left|l\left(\omega_{n}\right)\right|+\left|l\left(\omega_{n+1}\right)\right|\right)\right\}
$$

(with the lower bound $=0$ for $n=1$ ). Then $U_{n}$ is a sequence of disjoint, $S^{\beta}$. invariant, open sets in $\Omega$ with $\omega_{n} \in U_{n}$. For each $n$, pick an $f_{n} \in C_{0}(\Omega)$ such that $f$ has support in $U_{n},\left\|f_{n}\right\|=n^{-2}$ and $f_{n} \notin D\left(S_{\beta}\right)$. (The last condition is possible since $U_{n}$ contains nonfixed points for $S^{\beta}$.) Then $f=\sum_{n=1}^{\infty} f_{n} \in$ $C_{0}(\Omega)$, and $f \notin D\left(\delta_{\beta}\right)$ since $\delta_{\beta}$ coincides with differentiation along $S_{\beta}$ [B, Lemma 2.4.3]. Put $g_{n}=e^{-\left|\delta_{\beta}\right|} f_{n}$ and $g=e^{-\left|\delta_{\beta}\right|} f$. As $e^{-\left|\delta_{\beta}\right|}$ is a contraction semigroup, we have $g=\sum_{n=1}^{\infty} g_{n}$. As

$$
g_{n}(\omega)=\frac{1}{\pi} \int_{-\infty}^{\infty} \frac{d s}{1+s^{2}} f_{n}\left(S_{s}^{\beta} \omega\right)
$$

it follows that $g_{n}$, together with all its $\delta_{\beta}$-derivatives, are supported by the $S^{\beta}$-invariant set $U_{n}$, and thus

$$
\delta_{\beta}^{m} g_{n}(\omega)= \begin{cases}\delta_{\beta}^{m} g(\omega) & \text { for } \omega \in U_{n}, \\ 0 & \text { otherwise }\end{cases}
$$

Hence

$$
\left|\delta_{\beta}^{m} g(\omega)\right| \geq\left|\delta_{\beta}^{m} g_{n}(\omega)\right|
$$

for all $m=0,1, \ldots, \omega \in \Omega$. These derivatives really exist, since $e^{-t\left|\delta_{\beta}\right|}$ is a holomorphic semigroup. More specifically, the function $z \rightarrow e^{-z\left|\delta_{\beta}\right|} x$ has a holomorphic extension to the right haifplane $\operatorname{Re} z>0$ for each $x \in \mathfrak{A}$, as is easily seen from the Poisson kernel representation. It follows from Lemma 4.2 that $g_{n}$ is analytic for $\delta_{\beta}$ with the radius of convergence of

$$
\sum_{m=0}^{\infty} \frac{t^{m}}{m !}\left\|\delta_{\beta}^{m} g_{n}\right\|
$$

at least one. But as $f_{n}$ is nonanalytic, the radius of convergence is exactly one. The same applies to $g$ and $f$. Now, putting

$$
l_{n}=\frac{1}{2}\left(\left|l\left(\omega_{n}\right)\right|+\left|l\left(\omega_{n-1}\right)\right|\right)
$$


for $n=2,3, \ldots$, we have

$$
\begin{aligned}
\sum_{m=0}^{\infty} \frac{t^{m}}{m !}\left\|\delta_{\alpha}^{m} g\right\| & =\sum_{m=0}^{\infty} \frac{t^{m}}{m !}\left\|l^{m} \delta_{\beta}^{m}(g)\right\| \\
& \geq \sum_{m=0}^{\infty} \frac{t^{m}}{m !}\left\|l^{m} \delta_{\beta}^{m}\left(g_{n}\right)\right\| \geq \sum_{m=0}^{\infty} \frac{\left(t l_{n}\right)^{m}}{m !}\left\|\delta_{\beta}^{m}\left(g_{n}\right)\right\|
\end{aligned}
$$

and the latter series diverges for $t l_{n}>1, n=2,3, \ldots$. As $l_{n} \uparrow+\infty$, it follows that the series diverges for all $t>0$. We conclude that $g \notin \mathfrak{A}_{\omega}^{\alpha}$ and thus $\mathfrak{A}_{\omega}^{\beta} \nsubseteq \mathfrak{A}_{\omega}^{\alpha}$. This contradiction establishes that $l$ is bounded, and $(3) \Rightarrow(2)^{\prime \prime}$ follows.

In order to prove $(1) \Rightarrow(1)^{\prime \prime} \Rightarrow(2)^{\prime \prime}$ we need some terminology from [BER], or [B, Definition 2.4.17]. For $\omega \in \Omega$, let $\nu(\omega)=\nu_{\beta}(\omega) \in[0, \infty]$ be the frequency of $\omega$ with respect to $S^{\beta}$. The function $\omega \rightarrow \nu(\omega)$ is then upper semicontinuous, so the sets $\{\omega \in \Omega ; \nu(\omega)<a\}$, where $a \in[0, \infty]$, are open.

Assume (1). It follows that

$$
\mathfrak{A}_{F}^{\beta}=\bigcup_{L>0} \mathfrak{A}^{\beta}([-L, L]) \subseteq \mathfrak{A}_{F}^{\alpha} \subseteq D\left(\delta_{\alpha}\right) .
$$

Again using [KR, Theorem 2.3] there exists a continuous function $l$ on $\Omega \backslash \Omega_{0}$, which is constant on $S^{\beta}$-orbits and bounded on sets of bounded frequency, such that

$$
\left(\delta_{\alpha} f\right)(\omega)=l(\omega)\left(\delta_{\beta} f\right)(\omega) .
$$

Thus, the $S^{\beta}$-orbit through $\omega$ is also an $S^{\alpha}$-orbit, unless $l(\omega)=0$, in which case $\omega$ is a fixed point for $S^{\alpha}$, and the frequencies are related by $\nu_{\alpha}(\omega)=$ $l(\omega) \nu_{\beta}(\omega)$. Now, consider a particular $S^{\beta}$-orbit through $\omega$ with frequency $0<\nu_{\beta}(\omega)<\infty$. Then the orbit through $\omega$ and the flow $S^{\beta}$ restricted to this orbit is homeomorphic to $\mathbf{T}$ and the flow given by multiplication by $e^{i t}$ through

$$
S_{t}^{\beta} \omega \rightarrow e^{2 \pi i \nu_{\beta}(\omega) t} .
$$

Similarly the flow $S^{\alpha}$ restricted to this orbit is homeomorphic to $\left(\mathbf{T}, e^{i t}\right)$ through

$$
S_{t}^{\alpha} \omega \rightarrow e^{2 \pi i l(\omega) \nu_{\beta}(\omega) t} .
$$

Thus, if $L>0$ is given, the restrictions of the functions in $\mathfrak{A}^{\beta}([-L, L])$ to this orbit are linear combinations of the functions $z \in \mathbf{T} \Rightarrow z^{n}$, where $2 \pi \nu_{\beta}(\omega)|n| \leq$ $L$, while the restrictions of the functions in $\mathfrak{A}^{\alpha}([-c L-d, c L+d])$ to this orbit are linear combinations of the functions $z \in \mathbf{T} \Rightarrow z^{n}$, where

$$
2 \pi|l(\omega)| \nu_{\beta}(\omega)|n| \leq c L+d .
$$

By regularizing with functions whose Fourier transforms have suitable support properties, one sees that there really exist functions in the appropriate spectral 
subspaces for $\beta$ and $\alpha$ respectively, which restrict to the functions $z \rightarrow z^{n}$ on this particular orbit. The inclusion

$$
\mathfrak{A}^{\beta}([-L, L]) \subseteq \mathfrak{A}^{\alpha}([-c L-d, c L+d])
$$

for all $L>0$ thus implies

$$
2 \pi \nu_{\beta}(\omega) n \leq L \Rightarrow 2 \pi|l(\omega)| \nu_{\beta}(\omega) n \leq c L+d
$$

for $n=0,1,2, \ldots$ and all $L>0$. It follows that $|l(\omega)| \leq c$ and this again implies the stronger implication

$$
2 \pi \nu_{\beta}(\omega) n \leq L \rightarrow 2 \pi|l(\omega)| \nu_{\beta}(\omega) n \leq c L
$$

for $n=0,1,2, \ldots$ and all $L>0$.

Next, an $S^{\beta}$-orbit through $\omega$ with frequency $\nu_{\beta}(\omega)=0$ corresponds to the additive flow of $\mathbf{R}$ on $\mathbf{R}$, and if $S^{\beta}$ is flow with unit speed, $S^{\alpha}$ is flow with speed $l(\omega)$. Using continuous Fourier analysis one again exploits

$$
\mathfrak{A}^{\beta}([-L, L]) \subseteq \mathfrak{A}^{\alpha}([-c L-d, c L+d])
$$

to deduce that

$$
2 \pi|k| \leq L \Rightarrow 2 \pi|l(\omega)||k| \leq c L+d
$$

for all $k \in \mathbf{R}, L>0$. Hence $|l(\omega)| \leq c$ and therefore we obtain the stronger implication

$$
2 \pi|k| \leq L \rightarrow 2 \pi|l(\omega)||k| \leq c L .
$$

Thus $\|l\|_{\infty} \leq c$ and $\mathfrak{A}^{\beta}([-L, L]) \subseteq \mathfrak{A}^{\alpha}([-c L, c L])$ for all $L>0$. This ends the proof of $(1) \Rightarrow(1)^{\prime \prime} \Rightarrow(2)^{\prime \prime}$, and the remarks at the end of Proposition 4.1 follow from the proof.

\section{INFINITELY DIFFERENTIABLE ELEMENTS}

Proposition 2.1 indicates that the spaces of analytic element $\mathfrak{A}_{\omega}^{\alpha}, \mathfrak{A}_{\omega}^{\beta}$ are a fine gauge of the relative size of $\delta_{\alpha}, \delta_{\beta}$. In this section we show by examples that the spaces of $C^{\infty}$-elements $\mathfrak{A}_{\infty}^{\alpha}, \mathfrak{A}_{\infty}^{\beta}$ give a much cruder gauge. For Hilbert spaces this is immediate.

Proposition 5.1. Let $\mathfrak{A}$ be a Hilbert space and $\alpha, \beta$ a commuting pair of strongly continuous unitary one-parameter groups with generators $\delta_{\alpha}=i H_{\alpha}, \delta_{\beta}=i H_{\beta}$. The following two conditions are equivalent:

(1) $\mathfrak{A}_{\infty}^{\beta} \subseteq \mathfrak{A}_{\infty}^{\alpha}$.

(2) There exist constants $K, n$ such that $\left\|H_{\alpha} \psi\right\| \leq K\left(\left\|H_{\beta}^{n} \psi\right\|+\|\psi\|\right)$ for all $\psi \in \mathfrak{A}_{\infty}^{\alpha, \beta}$.

Proof. (2) $\Rightarrow(1)$ is immediate, while (1) $\Rightarrow(2)$ follows by spectral theory.

Thus, for Hilbert spaces $\mathfrak{A}_{\infty}^{\alpha}=\mathfrak{A}_{\infty}^{\beta}$ if and only if $H_{\alpha}$ and $H_{\beta}$ are mutually polynomially bounded, while $\mathfrak{A}_{\omega}^{\alpha}=\mathfrak{A}_{\omega}^{\beta}$ if and only if $H_{\alpha}$ and $H_{\beta}$ are mutually linearly bounded. 
Similarly, if $\mathfrak{A}$ is an abelian $C^{*}$-algebra, and $\alpha, \beta$ are one-parameter groups of ${ }^{*}$-automorphisms, it may happen that $\mathfrak{A}_{\infty}^{\alpha}=\mathfrak{A}_{\infty}^{\beta}$ even though $\alpha$ and $\beta$ are not mutually linearly bounded. An example [BER] is the $C^{*}$-algebra of continuous functions on the unit disc in $\mathbf{R}^{2}$, with

$$
\delta_{\alpha}=r^{-2} \partial / \partial \varphi, \quad \partial_{\beta}=r^{-1} \partial / \partial \varphi
$$

in polar coordinates. Then $\delta_{\alpha}=r^{-1} \partial_{\beta}$ so $\delta_{\alpha}$ is not linearly bounded by $\delta_{\beta}$. But $\partial_{\beta}^{2}=r^{-2} \partial^{2} / \partial \varphi^{2}$, and since

$$
\left\|\frac{\partial}{\partial \varphi} f\right\|_{\infty} \leq \varepsilon\left\|\frac{\partial^{2}}{\partial \varphi^{2}} f\right\|+\frac{2}{\varepsilon}\|f\|_{\infty}
$$

over each orbit for $\partial / \partial \varphi$, we have

$$
\left\|\delta_{\alpha} f\right\|_{\infty}=\left\|r^{-2} \frac{\partial}{\partial \varphi} f\right\|_{\infty} \leq \varepsilon\left\|r^{-2} \frac{\partial^{2}}{\partial \varphi^{2}} f\right\|+\frac{2}{\varepsilon}\left\|r^{-2} f\right\|_{\infty} \leq \varepsilon\left\|\delta_{\beta}^{2} f\right\|+\frac{2}{\varepsilon}\left\|r^{-2} f\right\|_{\infty} .
$$

This estimate may diverge since $f$ may be constant but replacing $f$ by

$$
g\left(r e^{i \varphi}\right)=f\left(r e^{i \varphi}\right)-f(r)
$$

on each orbit, we have $\|g\|_{\infty} \leq \pi\|\partial g / \partial \varphi\|_{\infty}$ on each orbit, i.e.,

$$
\left\|r^{-2} g\right\|_{\infty} \leq \pi\left\|r^{-2} \frac{\partial}{\partial \varphi} g\right\|_{\infty}=\pi\left\|\delta_{\alpha} g\right\|_{\infty}
$$

so we find in general (even when $f(r) \neq 0$ )

$$
\left\|\delta_{\alpha} f\right\|_{\infty} \leq \varepsilon\left\|\delta_{\beta}^{2} f\right\|+\frac{2 \pi}{\varepsilon}\left\|\delta_{\alpha} f\right\|_{\infty} .
$$

Optimizing this estimate with respect to $\varepsilon$, with $\varepsilon>2 \pi$, we find $\varepsilon=4 \pi$ and

$$
\left\|\delta_{\alpha} f\right\|_{\infty} \leq 8 \pi\left\|\delta_{\beta}^{2} f\right\| \text {. }
$$

On the other hand, $\left\|\delta_{\beta} f\right\|=\left\|r \delta_{\alpha} f\right\| \leq\left\|\delta_{\alpha} f\right\|$ so $\delta_{\alpha}$ and $\delta_{\beta}$ are relatively polynomially bounded, and thus $\mathfrak{A}_{\infty}^{\alpha}=\mathfrak{A}_{\infty}^{\beta}$.

In the opposite direction, if $\mathfrak{A}$ is prime, and $\beta$ is sufficiently "free" then all derivations $\delta$ defined on $\mathfrak{A}_{\infty}^{\beta}$ are a bounded perturbation of a scalar multiple of $\delta_{\beta}$, even without assuming that $\delta$ commutes with $\delta_{\beta}$ (see [B] for several examples). Thus $\mathfrak{A}_{\infty}^{\beta}$ determines $\delta$ even up to more than linear estimates. The assumption of freeness is essential for this, however, and we will next investigate a simple $C^{*}$-algebra and an automorphism group $\beta$ where the bounded perturbation property is not fulfilled. This example, originally investigated in [KR], is a product type action of $\mathbf{R}$ (or $\mathbf{T}$ ) on a UHF algebra: Let $M_{2}$ denote the algebra of complex $2 \times 2$ matrices, and let $\mathfrak{A}=\bigotimes^{\infty} M_{2}$ be the infinite tensor product of a countable number of copies of $M_{2}$. Further let $\alpha_{t}^{(n)}$ be 
the one-parameter group of automorphisms of $M_{2}$ implemented by the unitary group

$$
U_{t}^{n}=\left(\begin{array}{cc}
1 & 0 \\
0 & \exp \left\{-i \lambda_{n}(\alpha) t\right\}
\end{array}\right)
$$

where $\lambda_{n}(\alpha) \in \mathbf{R}$, and let $\alpha_{t}=\bigotimes_{n=1}^{\infty} \alpha_{t}^{n}$. Then there exists an $\alpha$-invariant pure state on $\mathfrak{A}$, and hence any derivation $\delta$ on $\mathfrak{A}_{\infty}^{\alpha}$ is a bounded perturbation of an invariant derivation, and thus a pregenerator, [K], [B, Theorem 2.9.10 and Theorem 2.6.8]. The derivation is not necessarily a bounded perturbation of a scalar multiple of a generator $\delta_{\alpha}$ of $\alpha$, however, but this is the case if $\Gamma(\alpha) \neq\{0\}$, where $\Gamma(\alpha)$ is the $\Gamma$-spectrum of $\alpha$ (the intersections of the closures of the sets

$\left\{\sum_{n=k}^{\infty} \varepsilon_{n} \lambda_{n}(\alpha) \mid \varepsilon_{n}= \pm, 0, \varepsilon_{n}=0\right.$ except for a finite number of $\left.n\right\}, \quad k \geq 1$,

see [KR] or [B, Theorem 2.6.6]). We thus have that $\Gamma(\alpha)=0$ if $\lambda_{n}(\alpha)$ grows rapidly, for example, if

$$
\lambda_{n}(\alpha)>\sum_{k=1}^{n-1}\left|\lambda_{k}(\alpha)\right|
$$

for all $n$. If in this situation $\delta$ is a derivation defined on $\mathfrak{A}_{\infty}^{\alpha}$, then $\delta$ is a bounded perturbation of a derivation $\delta_{\beta}$ commuting with $\delta_{\alpha}$, and, considering the spectral subspaces of $\delta_{\alpha}, \delta_{\beta}$ necessarily has the same form as $\delta_{\alpha}$, with $\lambda_{n}(\alpha)$ replaced by real numbers $\lambda_{n}(\beta)$. (Use that $\mathfrak{A}^{\alpha}$ is abelian, and hence $\delta_{\beta} \mid \mathfrak{A}^{\alpha}=0$.)

Example 5.2. The real numbers $\lambda_{n}(\alpha), \lambda_{n}(\beta)$ can be chosen positive and such that there exists a constant $C$ such that $\left\|\delta_{\beta}(x)\right\| \leq C\left\|\delta_{\alpha}(x)\right\|$ and $\left\|\delta_{\alpha}(x)\right\| \leq$ $C\left\|\delta_{\beta}^{2}(x)\right\|$ for all $x \in \mathfrak{A}_{\infty}^{\alpha}$, but there is no estimate of the form

$$
\left\|\delta_{\alpha}(x)\right\| \leq K\left(\left\|\delta_{\beta}(x)\right\|+\|x\|\right) .
$$

In this example $\mathfrak{A}_{\infty}^{\alpha}=\mathfrak{A}_{\infty}^{\beta}$ but $\mathfrak{A}_{\omega}^{\alpha} \varsubsetneqq \mathfrak{A}_{\omega}^{\beta}$.

Proof. Let $M$ be a positive number which is so large that $1-1 / M-1 / 4 M^{2}>0$ and $N / 2 M<1$ where $N$ is the constant in Lemma 2.4 of [KR].

We now choose the sequences $\lambda_{k}(\beta)>0, \lambda_{k}(\alpha)>0$ such that

(i) $\lambda_{k}(\alpha) \geq 2^{k+1} M \sum_{j=1}^{k} \lambda_{j}(\beta)$,

(ii) $\lambda_{k}(\beta)^{2} \geq 2^{k} \sum_{j=1}^{k} \lambda_{j}(\alpha)$,

(iii) $\lambda_{k}(\alpha) \geq 2 M \sum_{j=1}^{k-1} \lambda_{j}(\alpha)$,

(iv) $\lambda_{k}(\beta) \geq 2 M \sum_{j=1}^{k-1} \lambda_{j}(\beta)$.

This can be done by putting $\lambda_{1}(\beta)=1$ and then choosing $\lambda_{1}(\alpha), \lambda_{2}(\beta)$, $\lambda_{2}(\alpha), \lambda_{3}(\beta), \lambda_{3}(\alpha), \ldots$ inductively as follows: When all numbers up to $\lambda_{k-1}(\alpha)$ have been chosen, choose $\lambda_{k}(\beta)$ so large that (iv) is satisfied and such that (i) and (ii) are compatible, i.e., one can find a $\lambda_{k}(\alpha)$ such that (i) and (ii) both can 
be satisfied. By possibly choosing $\lambda_{k}(\beta)$ even larger, one can also assure that (iii) is satisfied for the $\lambda_{k}(\alpha)$ chosen in an interval allowed by (i) and (ii).

Having chosen the sequences $\lambda_{k}(\alpha), \lambda_{k}(\beta)$ one now derives the estimate $\left\|\delta_{\beta}(x)\right\| \leq C\left\|\delta_{\alpha}(x)\right\|$ from (i) and (iii) exactly as in [KR, pp. 90-93]. For the other estimate, we use the same technique: Let $\sigma_{n}^{ \pm}$be the matrices in the $n$th factor of $\mathfrak{A}=\bigotimes^{\infty} M_{2}$ defined by

$$
\sigma_{n}^{+}=\left(\begin{array}{ll}
0 & 1 \\
0 & 0
\end{array}\right), \quad \sigma_{n}^{-}=\left(\begin{array}{ll}
0 & 0 \\
1 & 0
\end{array}\right) .
$$

Then any element $x$ in $\mathfrak{A}_{0}$, the infinite algebraic tensor product of the factors $M_{2}$, can be written as a polynomial in $\sigma_{n}^{+}, \sigma_{n}^{-}$for $n=1,2, \ldots$ with coefficients in the fixed point algebra for $\alpha$ and $\beta$, which is the tensor product of the diagonal algebras $\left\{\left(\begin{array}{ll}a & 0 \\ 0 & b\end{array}\right) ; a, b \in \mathbf{C}\right\}$, and this decomposition in monomials is just the spectral decomposition of $x$ with respect to $\alpha$ or $\beta$ (here the rapid growth conditions (iii) and (iv) are essential). If $x_{n}^{+}$, respectively $x_{n}^{-}$, denotes the sum of those monomials which contain a factor $\sigma_{n}^{+}$, respectively $c_{n}^{-}$, but no factor $\sigma_{j}^{ \pm}$with $j>n$, then $x_{n}^{+}$is the $\alpha$-spectral component of $x$ corresponding to the spectral interval

$$
\left[\lambda_{n}(\alpha)-\sum_{j=1}^{k-1} \lambda_{j}(\alpha), \lambda_{n}(\alpha)+\sum_{j=1}^{k-1} \lambda_{j}(\alpha)\right] \subseteq\left[\left(1-\frac{1}{2 M}\right) \lambda_{n}(\alpha),\left(1+\frac{1}{2 M}\right) \lambda_{n}(\alpha)\right]
$$

and $x_{n}^{-}$the $\alpha$-spectral component corresponding to

$$
\left[-\left(1+\frac{1}{2 M}\right) \lambda_{n}(\alpha),-\left(1-\frac{1}{2 M}\right) \lambda_{n}(\alpha)\right] .
$$

All these intervals are disjoint because of (iii) and $1-1 / M-1 / 4 M^{2}>0$, and there is an $\varepsilon>0$ such that the distance between any two of these intervals is greater than $\varepsilon$. Similar remarks apply to $\beta$.

Now if $x \in \mathfrak{A}_{0}$, we can $\beta$-spectrally decompose $x$ as $x=x_{n}^{+}+\left(x-x_{n}^{+}\right)$ where the $\beta$-spectrum of $x_{n}^{+}$is contained in

$$
\left[\left(1-\frac{1}{2 M}\right) \lambda_{n}(\beta),\left(1+\frac{1}{2 M}\right) \lambda_{n}(\beta)\right]
$$

and the $\beta$-spectrum of $x-x_{n}^{+}$has distance at least $\varepsilon$ to this interval. The same remark applies to the decomposition

$$
\delta_{\beta}(x)=\delta_{\beta}\left(x_{n}^{+}\right)+\delta_{\beta}\left(x-x_{n}^{+}\right) .
$$

By Lemma 2.5 of $[\mathrm{KR}]$ there exists a $C$, only depending on $\varepsilon$, such that

$$
\left\|\delta_{\beta}\left(x_{n}^{+}\right)\right\| \leq C\left\|\delta_{\beta}(x)\right\|
$$

and by Lemma 2.4 of [KR],

$$
\left\|\delta_{\beta}\left(x_{n}^{+}\right)-i \lambda_{n}(\beta) x_{n}^{+}\right\|<N \frac{1}{2 M} \lambda_{n}(\beta)\left\|x_{n}^{+}\right\| .
$$


Combining these two estimates, we find

$$
\left\|\delta_{\beta}(x)\right\| \geq \lambda_{n}(\beta) C^{-1}\left(1-\frac{N}{2 M}\right)\left\|x_{n}^{+}\right\| .
$$

Applying this estimate on $\delta_{\beta}(x)$ instead of $x$ we get

$$
\left\|\delta_{\beta}^{2}(x)\right\| \geq \lambda_{n}(\beta) C^{-1}\left(1-\frac{N}{2 M}\right)\left\|\delta_{\beta}(x)_{n}^{+}\right\|,
$$

but as $\delta_{\beta}(x)_{n}^{+}=\delta_{\beta}\left(x_{n}^{+}\right)$and $\left(x_{n}^{+}\right)_{n}^{+}=x_{n}^{+}$we get

$$
\left\|\delta_{\beta}^{2}(x)\right\| \geq \lambda_{n}(\beta)^{2} C^{-2}\left(1-\frac{N}{2 M}\right)^{2}\left\|x_{n}^{+}\right\| .
$$

On the other hand, applying Lemma 2.4 of [KR] to $\delta_{\alpha}$, and using the fact that the $\alpha$-spectrum of $x_{n}^{+}$is contained in $\left[-\sum_{k=1}^{n} \lambda_{k}(\alpha), \sum_{k=1}^{n} \lambda_{k}(\alpha)\right]$, we then find

$$
\left\|\delta_{\alpha}\left(x_{n}^{+}\right)\right\| \leq N\left(\sum_{k=1}^{n} \lambda_{k}(\alpha)\right)\left\|x_{n}^{+}\right\| .
$$

Combining these two estimates, and using (ii), we next obtain

$$
\begin{aligned}
\left\|\delta_{\alpha}\left(x_{n}^{+}\right)\right\| & \leq C^{2}\left(1-\frac{N}{2 M}\right)^{-2} N \frac{\sum_{i=1}^{n} \lambda_{k}(\alpha)}{\lambda_{n}(\beta)^{2}}\left\|\delta_{\beta}^{2}(x)\right\| \\
& \leq C^{2}(1-N / 2 M)^{-2} N 2^{-n}\left\|\delta_{\beta}^{2}(x)\right\|=D 2^{-n}\left\|\delta_{\beta}^{2}(x)\right\|
\end{aligned}
$$

where the constant $D$ is independent of $x$ and $n$. Similarly,

$$
\left\|\delta_{\alpha}\left(x_{n}^{-}\right)\right\| \leq D 2^{-n}\left\|\delta_{\beta}(x)\right\|
$$

and since $x$ has the decomposition

$$
x=x_{0}+\sum_{n \geq 1} x_{n}^{+}+\sum_{n \geq 1} x_{n}^{-}
$$

we conclude that

$$
\begin{aligned}
\left\|\delta_{\alpha}(x)\right\| & \leq \sum_{n \geq 1}\left\|\delta_{\alpha}\left(x_{n}^{+}\right)\right\|+\sum_{n \geq 1}\left\|\delta_{\alpha}\left(x_{n}^{-}\right)\right\| \\
& \leq 2 D\left\|\delta_{\beta}^{2}(x)\right\| \sum_{n=1}^{\infty} 2^{-n}=2 D\left\|\delta_{\beta}^{2}(x)\right\| .
\end{aligned}
$$

This estimate is now valid for $x \in \mathfrak{A}_{0}$, but as $\mathfrak{A}_{0}$ is invariant under $\beta$, and even under $\exp \left(t \delta_{\beta}^{2}\right)$, it follows that $\mathfrak{A}_{0}$ is a core for $\delta_{\beta}^{2}$ [BR, Corollary 3.1.7]. Hence the above estimate extends by continuity to $x \in D\left(\delta_{\beta}^{2}\right)$.

This establishes the estimates of Example 5.2. Since

$$
\lim _{n \rightarrow \infty}\left(\lambda_{n}(\alpha) / \lambda_{n}(\beta)\right)=+\infty
$$

by (i), it is clear that we cannot have a linear estimate of the form

$$
\left\|\delta_{\alpha}(x)\right\| \leq K\left(\left\|\delta_{\beta}(x)\right\|+\|x\|\right)
$$


(in fact, $\left.\delta_{\alpha}\left(\sigma_{n}^{+}\right)=i \lambda_{n}(\alpha) \sigma_{n}^{+}, \delta_{\beta}\left(\sigma_{n}^{+}\right)=i \lambda_{n}(\beta) \sigma_{+}^{n}\right)$. Furthermore, since $\delta_{\alpha}$ and $\delta_{\beta}$ commute strongly, the joint $C^{\infty}$-vectors for $\alpha$ and $\beta, \mathfrak{A}_{\infty}^{\alpha, \beta}$, is a joint core for $\delta_{\alpha}^{n}$ and $\delta_{\beta}^{n}$ for all $n$, and for $x \in \mathfrak{A}_{\infty}^{\alpha, \beta}$

$$
\left\|\delta_{\beta}^{2}(x)\right\| \leq C\left\|\delta_{\alpha} \delta_{\beta} x\right\|=C\left\|\delta_{\beta} \delta_{\alpha} x\right\| \leq C^{2}\left\|\delta_{\alpha}^{2} x\right\|
$$

and by induction, $\left\|\delta_{\beta}^{n}(x)\right\| \leq C^{n}\left\|\delta_{\alpha}^{n}(x)\right\|$. Similarly, $\left\|\delta_{\alpha}^{n}(x)\right\| \leq C^{n}\left\|\delta_{\beta}^{2 n}(x)\right\|$, and from these two estimates it is clear that $\mathfrak{A}_{\infty}^{\alpha}=\mathfrak{A}_{\infty}^{\beta}$. Also, if $x$ is analytic for $\alpha, x$ is analytic for $\beta$ by Proposition $2.1, \mathfrak{A}_{\omega}^{\alpha} \subseteq \mathfrak{A}_{\omega}^{\beta}$. The other estimate above implies that analytic elements for $\beta$ are merely semianalytic for $\alpha$, and by an explicit calculation one can construct an element $x=\sum_{n \geq 1} c_{n} \sigma_{n}^{+}$which is analytic for $\beta$, but not for $\alpha$. This finishes the proof of the statements of Example 5.2.

\section{ONE-PARAMETER SEMIGROUPS OF CONTRACTIONS}

We now return to the situation considered in Lemma 4.2. The following example shows that Proposition 2.1 does not extend to the setting of contraction semigroups.

Example 6.1. Let $\mathfrak{A}=C_{0}(\mathbf{R})$ and $\delta=d / d x$. Then $\delta$ is not linearly bounded by $|\delta|$, and $|\delta|$ is not linearly bounded by $\delta$, even though $\delta$ and $|\delta|$ have the same analytic elements.

Proof. The operator $\delta$ generates the one-parameter groups of * -automorphisms given by $\left(e^{t \delta} f\right)(s)=f(s+t)$, and $f \in D(\delta)$ if and only if the derivative $f^{\prime}$ exists and $f^{\prime} \in C_{0}(\mathbf{R})$ [B, Lemma 2.4.3]. We then have

$$
\left(e^{-t|\delta|} f\right)(s)=\frac{t}{\pi} \int_{-\infty}^{\infty} \frac{d u}{t^{2}+u^{2}} f(s+u) .
$$

Now, let $f(t)=|t| g(t)$ for $|t|<1$, where

$$
g^{\prime}(t)=\frac{1}{t|\log | t||^{1+\varepsilon}}, \quad g(0)=0
$$

and $0<\varepsilon<1$, and extend $g$ so that $g$ is $C^{\infty}$ with compact support outside $[-1,1]$, and $g(t)=g(-t)$. This choice of $g^{\prime}$ ensures that $\int_{0}^{1} g^{\prime}(t) d t<+\infty$ so that $g$ is well-defined and continuous,

$$
\int_{0}^{1}|\log t| g^{\prime}(t) d t=\infty
$$

which we will use to show that $f \notin D(|\delta|)$, and

$$
f^{\prime}(t)=\frac{t}{|t|} g(t)+|t| g^{\prime}(t) \underset{t \rightarrow 0}{\longrightarrow} 0
$$

so that $f^{\prime}$ is continuous at $t=0$. 
Now, if $f \in D(|\delta|)$, the limit

$$
\begin{aligned}
-|\delta| f(0) & =\lim _{h \rightarrow 0} \frac{1}{h}\left(\left(e^{-h|\delta|} f\right)(0)-f(0)\right) \\
& =\lim _{h \rightarrow 0} \frac{h}{\pi h} \int_{-\infty}^{\infty} \frac{d s}{h^{2}+s^{2}} f(s)=\lim _{h \rightarrow 0} \frac{2}{\pi} \int_{0}^{\infty} \frac{d s}{h^{2}+s^{2}} s g(s)
\end{aligned}
$$

must exist. By partial integration,

$$
\frac{2}{\pi} \int_{0}^{\infty} \frac{s d s}{h^{2}+s^{2}} g(s)=-\frac{1}{\pi} \int_{0}^{\infty} \ln \left(h^{2}+s^{2}\right) g^{\prime}(s) d s .
$$

But as $\int_{0}^{1} \ln \left(s^{2}\right) g^{\prime}(s) d s$ diverges to $-\infty$, the monotone convergence theorem implies

$$
\lim _{h \rightarrow 0} \int_{0}^{\infty} \frac{d s}{h^{2}+s^{2}} s g(s)=-\infty
$$

and hence $f \notin D(|\delta|)$. Thus $|\delta|$ is not linearly bounded by $\delta$.

Conversely, if $f \in D\left(\delta^{2}\right)=D\left(|\delta|^{2}\right)$, we get by partial integration

$$
\begin{aligned}
\delta(1+|\delta|)^{-1} f & =\delta \int_{0}^{\infty} d t e^{-t} e^{-t|\delta|} f=\int_{0}^{\infty} d t e^{-t} \frac{t}{\pi} \int_{-\infty}^{\infty} \frac{d s}{t^{2}+s^{2}} \delta\left(e^{s \delta} f\right) \\
& =-\int_{0}^{\infty} d t e^{-t} \frac{t}{\pi} \int_{-\infty}^{\infty} d s \frac{d}{d s}\left(\frac{1}{t^{2}+s^{2}}\right) e^{s \delta} f \\
& =\int_{0}^{\infty} d t e^{-t} \frac{t}{\pi} \int_{-\infty}^{\infty} d s \frac{2 s}{\left(t^{2}+s^{2}\right)^{2}} e^{s \delta} f,
\end{aligned}
$$

so $\delta(1+|\delta|)^{-1}$ is a convolution operator with kernel

$$
\frac{2 s}{\pi} \int_{0}^{\infty} d t e^{-t} \frac{t}{\left(t^{2}+s^{2}\right)^{2}}=\frac{1}{\pi}\left(\frac{1}{s}-s \int_{0}^{\infty} \frac{d t e^{-t}}{t^{2}+s^{2}}\right) .
$$

But

$$
\left|s \int_{0}^{\infty} \frac{d t e^{-t}}{t^{2}+s^{2}}\right| \leq \int_{0}^{\infty} \frac{d u}{1+u^{2}}=\frac{\pi}{2}
$$

so the kernel differs from $1 / \pi s$ by a bounded function. Thus the kernel is nonintegrable, and since the norm of $\delta(1+|\delta|)^{-1}$ is equal to the $L^{1}$-norm of the kernel, it follows that $\delta(1+|\delta|)^{-1}$ is unbounded. Thus $\delta$ is not linearly bounded by $|\delta|$.

There is a final somewhat different example of two contraction semigroups whose generators have distinct domains but their $C^{\infty}$-elements coincide, and in addition their analytic elements coincide. This example is constructed with the aid of two Laplacians, $\Delta_{\alpha}, \Delta_{\beta}$, defined on $C_{0}^{\infty}\left(\mathbf{R}^{d}\right)$. These Laplacians are defined with respect to distinct coordinate bases. Now the closures, $\bar{\Delta}_{\alpha}, \bar{\Delta}_{\beta}$, of these operators generate continuous contraction semigroups $S^{\alpha}, S^{\beta}$, on $C_{0}\left(\mathbf{R}^{d}\right)$, but since there is no universal estimate

$$
\left\|\partial_{i} \partial_{j} f\right\|_{\infty} \leq C\left(\left\|\Delta_{\alpha} f\right\|+\|f\|\right)
$$


for all $f \in C_{0}^{\infty}\left(\mathbf{R}^{d}\right)$ (see [O]) it follows that $D\left(\bar{\Delta}_{\alpha}\right) \neq D\left(\bar{\Delta}_{\beta}\right)$. In fact, $D\left(\bar{\Delta}_{\beta}\right)=$ $D\left(\bar{\Delta}_{\beta}\right)$ only if $\Delta_{\alpha}$ and $\Delta_{\beta}$ are proportional. Nevertheless, the $C^{\infty}$-elements of $S^{\alpha}$ and $S^{\beta}$ coincide with $C_{0}^{\infty}\left(\mathbf{R}^{d}\right)$ and their analytic elements can be characterized as those $f \in C_{0}^{\infty}\left(\mathbf{R}^{d}\right)$ such that

$$
\sum_{n \geq 1} \frac{t^{n}}{n !}\|f\|_{2 n}<\infty,
$$

for some $t>0$, where $\|\cdot\|_{2 n}$ denotes the $C_{0}^{2 n}$-norm. This latter identification can be obtained by the use of Lipschitz space estimates as in $\S 6$ of [R2].

Acknowledgement. This work was begun while O. Bratteli was visiting the Australian National University in December 1987, and finished while the three authors were visiting the University of Iowa in June 1988. We would like to thank Fred Goodman and Palle Jørgensen for their hospitality and for discussions at the University of Iowa.

\section{REFERENCES}

[B] O. Bratteli, Derivations, dissipations and group actions on $C^{*}$-algebras, Lecture Notes in Math., vol. 1229, Springer-Verlag, Berlin and New York, 1986.

[BGR] O. Bratteli, T. Digernes, F. Goodman and D. W. Robinson, Integration in abelian $C^{*}$ dynamical systems, Publ. Res. Inst. Math. Sci. 21 (1985), 1001-1030.

[BER] O. Bratteli, G. A. Elliott and D. W. Robinson, The characterization of differential operators by locality: Classical flows, Compositio Math. 58 (1986), 279-319.

[BGJR] O. Bratteli, F. Goodman, P. E. T. Jørgensen and D. W. Robinson, The heat semigroup and integrability of Lie algebras, J. Funct. Anal. 79 (1988), 351-397.

[BR] O. Bratteli and D. W. Robinson, Operator algebras and quantum statistical mechanics. I (2nd ed.), Springer-Verlag, Berlin and New York, 1987.

[GJ] F. M. Goodman and P. E. T. Jørgensen, Lie algebras of unbounded derivations, J. Funct. Anal. 52 (1983), 369-384.

[K] A. Kishimoto, Derivations with a domain condition, Yokohama Math. J. 32 (1984), 215223.

[KR] A. Kishimoto and D. W. Robinson, Derivations, dynamical systems and spectral restrictions, Math. Scand. 56 (1985), 83-95.

[N] E. Nelson, Analytic vectors, Ann. of Math. 70 (1959), 572-615.

[O] D. Ornstein, A non-inequality for differential operators in the $L_{1}$-norm, Arch. Rational Mech. Anal. 11 (1962), 40-49.

[R1] D. W. Robinson, Differential and integral structure of continuous representations of Lie groups, J. Operator Theory 19 (1988), 95-128.

[R2] _ Lie groups and Lipschitz spaces, Duke Math. J. 57 (1988), 357-395.

[Y] K. Yosida, Functional analysis (6th ed.), Springer-Verlag, Berlin and New York, 1980.

Institute of Mathematics, University of Trondheim, N-7034 Trondheim NTH, NoRway

Department of Applied Mathematics, Faculty of Science, Fukuoka University, 8-19-1 Nanakuma, Jonanku, FukUoka 814-01, Japan

Mathematics Research Section-SMS, Australian National University-IaS, GPO Box 4, Canberra, ACT 2601, Australia 